Pacific Journal of Mathematic 


\title{
RETRACTION METHODS IN NIELSEN FIXED POINT THEORY
}

\author{
ROBERT F. BROWN
}

Let $X$ be a topological space, $A$ a subset of $X$, and $F: X \rightarrow X$ a map. Suppose there exists a retraction $\rho: W \rightarrow A$ where $A \cup F(A) \subseteq W \subseteq X$; then define $f: A \rightarrow A$ by $f(a)=\rho F(a)$. The map $f$ is called a retract of $F$. If all the fixed points of $f$ are fixed points of $F$, we say that $F$ is retractible onto $A$ (with respect to $\rho$ ). Then, if $A$ is a compact ANR, the Nielsen number $N(f)$ of $f$ is a lower bound for the number of fixed points of $F$, or of any map $G: X \rightarrow X$ retractible onto $A$ with retract homotopic to $f$. Many classes of examples of retractible maps can be found, even if $X$ is required to be a euclidean space. If $F$ is retractible onto a compact ANR with respect to a deformation retraction of $X$ onto $A$, then we say that $F$ is deformation retractible (dr) and we define a number $D(F)$ which we prove to have the property: if $G: X \rightarrow X$ is a dr map homotopic to $F$, then $G$ has at least $D(F)$ fixed points. If $X$ is an ANR and $F$ is a compact map, then $D(F)$ is the Nielsen number of $F$. We find conditions, for any map $F: X \rightarrow X$ retractible onto $A$, so that there exists $G: X \rightarrow X$ retractible onto $A$ and with retract homotopic to $f$ such that $G$ has exactly $N(f)$ fixed points. Furthermore, if $F$ is dr, the hypotheses yield a dr map $G$ homotopic to $F$ and with exactly $D(F)$ fixed points. These last results are based on a technique, of independent interest, for extending a map $g: A \rightarrow A$, on a finite subpolyhedron of a locally finite polyhedron $X$, to a map $G: X \rightarrow X$ in such a way that $G$ has no fixed points on $X-A$.

1. The Poincaré-Bohl theorem. Retraction-type results are among the oldest in fixed point theory. In 1904, Bohl [1] proved

THEOREM 1.1. Let $C=\left\{x=\left(x_{1}, \ldots, x_{n}\right) \in \mathbf{R}^{n}|| x_{l} \mid \leq a_{i}\right\}$ for some positive numbers $a_{1}, \ldots, a_{n}$. If $g: C \rightarrow \mathbf{R}^{n}-0$ is a map, then there exists $a$ point $x$ on the boundary of $C$ such that $g(x)=\alpha x$, for some $\alpha<0$.

In 1910, Hadamard [13] observed that Bohl's result was equivalent to an earlier theorem of Poincaré [28] and therefore called it the Poincaré-Bohl Theorem. It will be convenient to restate the theorem in the form:

Theorem 1.2. (Poincaré-Bohl Theorem.) Let $F: \mathbf{R}^{n} \rightarrow \mathbf{R}^{n}$ be a map and suppose there exists $R>0$ such that

$$
\|x\|=R \text { implies } F(x) \neq \lambda x, \quad \text { for all } \lambda>1 .
$$

Then $F(x)=x$ for some $x$ with $\|x\| \leq R$. 
It is not difficult to show that this statement is equivalent to Bohl's result.

To see the relationship of the Poincaré-Bohl theorem to the retraction concept, we give the following well-known proof (compare [22]). The proof also serves to introduce concepts that will be used in later sections. Set $B_{R}=\left\{x \in \mathbf{R}^{n} \mid\|x\| \leq R\right\}$ and define a "radial" retraction $\rho: \mathbf{R}^{n} \rightarrow B_{R}$ by

$$
\rho(x)= \begin{cases}\frac{R}{\|x\|} \cdot x & \text { if }\|x\| \geq R, \\ x & \text { if }\|x\| \leq R .\end{cases}
$$

Let $\iota: B_{R} \rightarrow \mathbf{R}^{n}$ be inclusion and define $f=\rho F \iota: B_{R} \rightarrow B_{R}$. By the Brouwer Fixed Point Theorem, $f(x)=x$ for some $x \in B_{R}$. It cannot be that $\|F(x)\|>R$ because then we would have $f(x)=R /\|F(x)\| \cdot F(x)=x$, or $F(x)=\|F(x)\| / R \cdot x$, contrary to $(*)$, so it must be that $F(x)=x$.

Letting "Fix" denote the set of fixed points of a map; we see from the argument that $(*)$ implies $\operatorname{Fix}(f)=\operatorname{Fix}(F) \cap B_{R}$. Thus, information about fixed points of $f$ is information about fixed points of $F$ as well. Notice that the use of the radial retraction $\rho$ permitted us to "retract" the map $F$ of $\mathbf{R}^{n}$ to a map $f$ on the compact space $B_{R}$ for which an appropriate fixed point theorem was available.

2. The basic example. In this section, we will extend the techniques of the above proof of the Poincaré-Bohl theorem to prove a result which will serve as a model for the approach we use in the rest of the paper.

Let $0<r<R$ be given and define the annulus

$$
A_{r, R}=\left\{x \in \mathbf{R}^{2} \mid r \leq\|x\| \leq R\right\} .
$$

Denote the degree of a map $f: A_{r, R} \rightarrow A_{r, R}$ by $\operatorname{deg}(f)$. In 1921, Brouwer [2] used a technique of Nielsen [27] to obtain a lower bound for the number of fixed points of a map from a torus $T=S^{1} \times S^{1}$ to itself. If we view $T$ as the double of $A_{r, R}$, we can use $f$ from both the "top half" and the "bottom half" to, say, the top half to define a map $f^{\prime}: T \rightarrow A_{r, R} \subseteq T$. It follows from the Nielsen-Brouwer theorem that $f^{\prime}$, and therefore the map $f$ as well, has at least $|\operatorname{deg}(f)-1|$ fixed points.

In place of the retraction $\rho: \mathbf{R}^{n} \rightarrow B_{R}$ we used in $\S 1$, we need the radial retraction $\rho: \mathbf{R}^{2}-0 \rightarrow A_{r, R}$ defined by

$$
\rho(x)= \begin{cases}\frac{r}{\|x\|} \cdot x & \text { if } 0<\|x\| \leq r, \\ x & \text { if } r \leq\|x\| \leq R, \\ \frac{R}{\|x\|} \cdot x & \text { if }\|x\| \geq R .\end{cases}
$$


Given a map $F: \mathbf{R}^{2} \rightarrow \mathbf{R}^{2}$ such that $F\left(A_{r, R}\right) \subseteq \mathbf{R}^{2}-0$, we can again retract $F$, this time to a map on $A_{r, R}$, by letting $\iota: A_{r, R} \rightarrow \mathbf{R}^{2}$ be inclusion and setting $f=\rho F_{\iota}: A_{r, R} \rightarrow A_{r, R}$.

Proposition 2.1. Let $F: \mathbf{R}^{2} \rightarrow \mathbf{R}^{2}$ be a map. Suppose there exist $0<r<R$ such that:

(0) $r \leq\|x\| \leq R$ implies $F(x) \neq 0$;

$(*)\|x\|=R$ implies $F(x) \neq \lambda x$, for all $\lambda>1$;

$(* *)\|x\|=r$ implies $F(x) \neq \mu x$, for all $0<\mu<1$.

Then $F$ has at least $|\operatorname{deg}(f)-1|$ fixed points $x$ with $r \leq\|x\| \leq R$.

Condition ( 0 ) is required so that $f$ will be well-defined. The NielsenBrouwer theorem implies that $f$ has at least $|\operatorname{deg}(f)-1|$ fixed points. Suppose $f(x)=x$; then $\|F(x)\|>R$ would contradict $(*)$, precisely as in $\S 1$. Similarly $\|F(x)\|<r$ would contradict $(* *)$. Therefore, conditions (*) and (**) imply that $\operatorname{Fix}(f)=\operatorname{Fix}(F) \cap A_{r, R}$, so $F$ has at least $|\operatorname{deg}(f)-1|$ fixed points, which lie in $A_{r, R}$. Obviously this result could have been proved at any time since 1921, but it seems not to have been noticed.

The Nielsen-Brouwer theorem, which is the basis for Proposition 2.1, depends on the fact that the fundamental group is not trivial. Thus, in this section, the advantage of retracting, and then studying a map on $A_{r, R}$ rather than on $\mathbf{R}^{2}$, is that not only are we working on a compact set, but on one that is non-simply-connected.

3. Retractibility. With the previous two sections in mind, it is natural to make the following definitions. Let $X$ be a topological space and let $F: X \rightarrow X$ be a map. Suppose for a subset $A$ of $X$ there is a retraction $\rho: W \rightarrow A$ where $A \cup F(A) \subseteq W \subseteq X$. Then, for $\iota: A \rightarrow X$ the inclusion, $f=\rho F \iota: A \rightarrow A$ is a well-defined map we call the retract of $F$ (with respect to $\rho$ ). The following easily proved result justifies our use of this terminology.

Proposition 3.1. A subspace $A$ of a space $X$ is a retract of $X$ if, and only if, the identity map on $A$ is a retract of the identity map on $X$.

The example we keep in mind is $X=\mathbf{R}^{2}, A=A_{r, R}, W=\mathbf{R}^{2}-0$, and $\rho$ is the radial retraction. A map $F: \mathbf{R}^{2} \rightarrow \mathbf{R}^{2}$ has a retract with respect to $\rho$ provided $F\left(A_{r, R}\right) \subseteq \mathbf{R}^{2}-0$.

Returning to the general setting, notice that even if a map $F: X \rightarrow X$ has a retract $f: A \rightarrow A$, the map $f$ is of no use in studying the fixed points of $F$ unless we require more. Thus, we call $F$ "retractible" onto $A$ only if 
the fixed points of the retract $f$ are related to those of $F$ as in the previous sections. To be precise, we say that $F: X \rightarrow X$ is retractible onto a subset $A$ of $X$ if there exists $W$ in $X$ containing $A \cup F(A)$ and a retraction $\rho$ : $W \rightarrow A$ such that $\operatorname{Fix}(f)=\operatorname{Fix}(F) \cap A$, for $f: A \rightarrow A$ the retract of $F$ with respect to $\rho$. The following simple observation is useful in verifying that a map is retractible onto a set.

Proposition 3.2. Let $f: A \rightarrow A$ be the retract of a map $F: X \rightarrow X$ with respect to a retraction $\rho: W \rightarrow A$, where $A \cup F(A) \subseteq W$. Then $\operatorname{Fix}(f)=$ $\operatorname{Fix}(F) \cap A$ if, and only if, for each $x \in \rho(F(A)-A)$, either $F(x)=x$ or $F(x) \notin \rho^{-1}(x)$.

Having replaced the hypotheses of the basic example, Proposition 2.1, by a suitable generalization, we need a form of the conclusion that is appropriate for spaces other than the annulus. For a map $f: A \rightarrow A$ on a compact ANR, the Nielsen number $N(f)$ is a lower bound for the number of fixed points of $f$. Homotopic maps have equal Nielsen numbers. The relationship with Proposition 2.1 is given by the fact that if $f: A \rightarrow A$ is a map on an annulus, then $N(f)=|\operatorname{deg}(f)-1|$. Expositions of Nielsen fixed point theory can be found in [4] and [21]. The generalization of Proposition 2.1 is

Proposition 3.3. Let $X$ be a topological space and let $A$ be a compact $A N R$ in $X$. Suppose a map $F: X \rightarrow X$ is retractible onto $A$, with retract $f$ : $A \rightarrow A$. If a map $G: X \rightarrow X$ is retractible onto $A$, with retract $g: A \rightarrow A$ homotopic to $f$, then $G$ has at least $N(f)$ fixed points in $A$.

REMARKS. (1) If $A$, a compact ANR, is simply-connected, then $N(f) \leq 1$ for any map $f: A \rightarrow A$. Thus Proposition 3.3 is of interest primarily when $F$ can be retracted to a map on a non-simply-connected set such as the annulus of $\S 2$.

(2) Since the retract $f$ depends only on the behavior of $F$ on $A$, the definition of retractibility and the statement of Proposition 3.3 could have been given in terms of maps $F: A \rightarrow X$. However, fixed point theory is generally concerned with the fixed points of a self-map of a space, so it seems more natural to assume $F: X \rightarrow X$ is such a map, of a space on which the usual topological methods cannot be used (for instance, when $X$ is a euclidean space). Then, if $F$ is retractible onto a compact ANR, Proposition 3.3 implies that we can, nevertheless, recover information about $\operatorname{Fix}(F)$ by those methods. 
(3) The approach suggested by 3.3 is also useful in nonlinear analysis; see [5].

In the next section, we present some examples of maps retractible onto compact ANRs. We restrict ourselves to maps on euclidean spaces to keep the list reasonably short.

Section 5 begins with an example that illustrates the fact that the lower bound $N(f)$ for the number of fixed points of $F: X \rightarrow X$ on $A$ depends on the choice of the retraction $\rho$. We can overcome this problem by restricting ourselves to retractions that, like the radial retractions of the first two sections, are deformation retractions. Then we prove that when we impose an additional restriction on the map $F$ as well, the lower bound depends only on the homotopy class of $F$ and therefore we can define a "Nielsen number" for such maps.

In the Nielsen fixed point theory of maps $f: K \rightarrow K$ on finite polyhedra, the Nielsen number $N(f)$ is not only a lower bound for the number of fixed points of $f$ or any map homotopic to it but, if the polyhedron $K$ satisfies certain additional topological conditions, then $N(f)$ is a sharp lower bound in the sense that $f$ can be homotoped to a map with exactly $N(f)$ fixed points. Sections 6 and 7 are devoted to an analogous result concerning locally finite polyhedra, for maps which are retractible onto finite polyhedra. The preliminary results of $\$ 6$ present a technique for extending a map $g: A \rightarrow A$, on a finite subpolyhedron of a locally finite polyhedron $X$, to a map $G: X \rightarrow X$ without introducing additional fixed points, that is, such that $\operatorname{Fix}(G)=\operatorname{Fix}(g)$. This technique is of interest independent of its use in proving the "minimality theorem", of $\S 7$.

4. Other examples of retractible maps. The distance between points $x, y \in \mathbf{R}^{n}$ is $\|x-y\|$, so for $C$ a subset of $\mathbf{R}^{n}$ and $x \in \mathbf{R}^{n}$ we have the "distance"

$$
d(x, C)=\inf \{\|x-y\| \mid y \in C\} .
$$

We can generalize the example of $\S 2$ as follows. Let $k>0$ be given and define $S \subseteq \mathbf{R}^{n}$ as the set of points $x=\left(x_{1}, \ldots, x_{n}\right)$ such that $x_{1}^{2}+x_{2}^{2}$ $=k^{2}$ and $x_{3}=\cdots=x_{n}=0$. Given $\varepsilon$ such that $0<\varepsilon<k$, let

$$
A=A(k, \varepsilon)=\left\{x \in \mathbf{R}^{n} \mid d(x, S) \leq \varepsilon\right\},
$$

then $A$ is a solid $n$-torus, that is, it is homeomorphic to $S^{1} \times B^{n-1}$. Define

$$
W=\left\{x=\left(x_{1}, \ldots, x_{n}\right) \in \mathbf{R}^{n} \mid x_{1}^{2}+x_{2}^{2} \neq 0\right\}
$$

then for each $x \in W$ there is a unique point $p(x) \in S$ such that $\| x-$ $p(x) \|=d(x, S)$. Let $\partial A$ denote the boundary of $A$. For each $x$ in $W-A$, 
the line segment between $x$ and $p(x)$ intersects $\partial A$ at one point; call it $\rho(x)$. Extend $\rho$ to all of $W$ by setting $\rho(x)=x$ for $x \in A$, then $\rho$ is continuous and therefore a retraction of $W$ onto $A$. Furthermore, $\| x-$ $\rho(x) \|=d(x, A)$ for all $x \in W$. If we take $n=2, k=\frac{1}{2}(R+r)$, and $\varepsilon$ $=\frac{1}{2}(R-r)$, then $W=\mathbf{R}^{2}-0$ and $\rho$ is radial retraction, so in this way we recover the example of $\S 2$.

A map $F: \mathbf{R}^{n} \rightarrow \mathbf{R}^{n}$ will be retractible onto $A=A(k, \varepsilon)$ with respect to the retraction $\rho$ if $F(A) \subseteq W$ and, for each $x \in \partial A$, either $F(x) \in A$ or, if $F(x) \notin A$, then $x$ does not lie on the line segment between $F(x)$ and $p F(x)$ because, if it did, then $x=\rho(F(x)$ ) (compare Proposition 3.2). In particular, a map $F: \mathbf{R}^{n} \rightarrow \mathbf{R}^{n}$ is retractible onto $A$ if $F(A) \subseteq W$ and $F(\partial A) \subseteq A$. We can replace the condition $F(\partial A) \subseteq A$ by a considerably weaker one, as follows. For each $x \in \partial A$, let

$$
I_{A}(x)=\{x+c(a-x) \mid a \in A \text { and } c \geq 1\} .
$$

Then, following Caristi [6], we say that $F$ is weakly inward on $\partial A$ if $F(x)$ is in the closure of $I_{A}(x)$, for all $x \in \partial A$.

Proposition 4.1. Let $A=A(k, \varepsilon)$ be a solid torus in $\mathbf{R}^{n}$ and let $W=\left\{\left(x_{1}, \ldots, x_{n}\right) \in \mathbf{R}^{n} \mid x_{1}^{2}+x_{2}^{2} \neq 0\right\}$. If $F: \mathbf{R}^{n} \rightarrow \mathbf{R}^{n}$ is a map such that $F(A) \subseteq W$ and $F$ is weakly inward on $\partial A$, then $F$ is retractible onto $A$.

Proof. Given $x \in \partial A$, let $u$ be a point of $W-A$ such that $\rho(u)=x$. We have observed above that this implies $\|u-x\|=d(u, A)$. We claim that if $z$ is a point such that $\|z-u\|<\|x-u\|$, then $z \notin I_{A}(x)$. To prove the claim, assume the contrary, so $z=x+c(a-x)$ for some $a \in A$ and $c \geq 1$. Then

$$
a=\frac{1}{c} z+\left(1-\frac{1}{c}\right) x
$$

and

$$
\|a-u\| \leq \frac{1}{c}\|z-u\|+\left(1-\frac{1}{c}\right)\|x-u\|<\|x-u\|=d(u, A),
$$

which is impossible because $a \in A$. We have established the claim and therefore $u$ is in an open subset of $\mathbf{R}^{n}$ disjoint from $I_{A}(x)$. Since $F$ is weakly inward on $\partial A$, we see that $F(x) \neq u$ for all $u \in W-A$ with $\rho(u)=x$, so $\operatorname{Fix}(f)=\operatorname{Fix}(F) \cap A$ by Proposition 3.2, where $f$ is the retract of $F$ with respect to $\rho$.

A map $F: \mathbf{R}^{n} \rightarrow \mathbf{R}^{n}$ satisfying the hypotheses of Proposition 4.1 has at least $|\operatorname{deg}(f)-1|$ fixed points, where $f$ is the retract of $F$ with respect to $\rho$, 
because, by a theorem of Hopf [14], $f$ has at least that many. This computation also follows from Proposition 3.3 and [18].

Observe that Proposition 4.1 generalizes to any sets $A \subseteq W \subseteq \mathbf{R}^{n}$ provided there is a "closest point" retraction $\rho: W \rightarrow A$, that is, one such that $\|x-\rho(x)\|=d(x, A)$ for all $x \in W$. The same proof shows that if $F: \mathbf{R}^{n} \rightarrow \mathbf{R}^{n}$ is a map with $F(A) \subseteq W$ and $F$ weakly inward on $\rho(W-A)$, then $F$ is retractible onto $A$.

The next class of examples was developed with the assistance of Robert Edwards. Let $A$ be a compact $n$-manifold with nonempty boundary $\partial A$ and suppose $A$ is embedded in $\mathbf{R}^{n}$ as a subpolyhedron. Write $S^{n}=\mathbf{R}^{n}$ $\cup\{\infty\}$ and let $Y$ be a complementary spine of $A$ in $S^{n}$, in the sense of Rourke [29]. That is, $Y$ is a subpolyhedron of $S^{n}-A$ such that $S^{n}-\AA$ collapses to $Y$, where $\AA=A-\partial A$. We will assume, without loss of generality, that $\infty \in Y$. The manifold $S^{n}-\AA$ is a regular neighborhood of $Y$. Let $N(Y)$ be the second derived regular neighborhood of $Y$ [17; page 50], then $N(Y)-Y$ is homeomorphic to $\partial N(Y) \times[0,1)$, so there is a retraction of $N(Y)-Y$ onto $\partial N(Y)$. Consequently, by the uniqueness of regular neighborhoods [30; page 33], $\left(S^{n}-\AA\right)-Y$ retracts to $\partial\left(S^{n}-\AA\right)$ $=\partial A$, by a retraction we will call $\rho$. Let $W=S^{n}-Y=R^{n}-Y$, then we can extend $\rho$ to a retraction $\rho: W \rightarrow A$.

In the previous example (Proposition 4.1), $A$ was a solid torus, the complementary spine in $S^{n}$ was $Y=\left\{\left(0,0, x_{3}, \ldots, x_{n}\right) \in \mathbf{R}^{n}\right\} \cup\{\infty\}$, which is homeomorphic to $S^{n-2}$, and we used a "closest point" retraction $\rho: \mathbf{R}^{n}-Y \rightarrow A$. Since the retraction obtained from the regular neighborhood structure on $S^{n}-\AA$ lacks the "closest point" property, the best we can do in general is the following.

Proposition 4.2. Let $A$ be a compact $n$-manifold with nonempty boundary $\partial A$ and suppose $A$ is embedded in $\mathbf{R}^{n}$ as a subpolyhedron. Let $Y$ be a complementary spine of $A$ in $S^{n}=\mathbf{R}^{n} \cup\{\infty\}$ such that $\infty \in Y$. If $F$ : $\mathbf{R}^{n} \rightarrow \mathbf{R}^{n}$ is a map such that $F(A) \cap Y=\varnothing$ and $F(\partial A) \subseteq A$, then $F$ is retractible onto $A$.

In view of the hypothesis $F(A) \cap Y=\varnothing$ in Proposition 4.2, it is desirable that we choose a complementary spine of $A$ in $S^{n}$ that is as "small" as possible. If $\pi_{i}\left(S^{n}-\AA, \partial A\right)=0$ for all $i \leq r$, where $r \leq n-4$, then it follows from a theorem of Wall [34] that $A$ has a complementary spine of dimension less than or equal to $n+1-r$.

We can combine 4.2 with Proposition 3.3 to obtain a lower bound for the number of fixed points of $F$, provided we can compute the Nielsen 
number of the retract $f: A \rightarrow A$. There is an extensive treatment of the computational problem, due to Jiang, available for this purpose [18], [20], [21].

Next we suppose that $A$ is still a compact $n$-manifold with nonempty boundary $\partial A$ and that $A$ is embedded in $\mathbf{R}^{n}$, but now we require that $A$ be a smooth $\left(C^{\infty}\right)$ submanifold of $\mathbf{R}^{n}$. Then $\partial A$ is a smooth $(n-1)$-manifold without boundary embedded in $\mathbf{R}^{n}$ so, by the local retraction theorem [26; page 51], there is a neighborhood $U$ of $\partial A$ in $\mathbf{R}^{n}$ and a retraction $\rho^{\prime}$ : $U \rightarrow \partial A$. Furthermore, from the proof of that theorem, we observe that if $u \in U$ and $v$ is in $T_{\rho^{\prime}(u)}(\partial A)$, the tangent space to $\partial A$ at $\rho^{\prime}(u)$, then $\left(\rho^{\prime}(u)-u\right) \cdot v=0$. Since the normal bundle of the embedding of $\partial A$ in $\mathbf{R}^{n}$ is trivial, the set $U$ is homeomorphic to an open subset of $\partial A \times \mathbf{R}$ by a homeomorphism $\varphi$ with the following property: let $\pi: \partial A \times \mathbf{R} \rightarrow \mathbf{R}$ be projection, then $\pi \varphi(u) \leq 0$ if and only if $u \in A$. Let

$$
W=A \cup\{u \in U \mid \pi \varphi(u)>0\}
$$

then $W$ is a neighborhood of $A$ in $\mathbf{R}^{n}$ and there is a retraction $\rho: W \rightarrow A$ defined by $\rho(x)=x$ if $x \in A$ and $\rho(x)=\rho^{\prime}(x)$ if $x \notin A$.

Proposition 3.2 and the property of $\rho^{\prime}$ noted above imply

Proposition 4.3. Let $A$ be a compact $n$-manifold with nonempty boundary $\partial A$ and suppose $A$ is embedded in $\mathbf{R}^{n}$ as a smooth submanifold. There is a neighborhood $W$ of $A$ in $\mathbf{R}^{n}$ such that if $F(A) \subseteq W$ and $(F(x)-x) \cdot v \neq 0$ for all $x \in \partial A$ and $v \in T_{x}(\partial A)$, then $F$ is retractible onto $A$.

The local retraction theorem of [26] can also be used if $A$ is a closed (that is, compact and without boundary) $k$-dimensional smooth submanifold of $\mathbf{R}^{n}$, for any $k<n$. In this case we have

Proposition 4.4. Let $A$ be a closed, smooth submanifold of $\mathbf{R}^{n}$. There is a neighborhood $W$ of $A$ in $\mathbf{R}^{n}$ such that if $F(A) \subseteq W$ and $(F(x)-x) \cdot v \neq 0$ for all $x \in A$ and $v \in T_{x}(A)$, then $F$ is retractible onto $A$.

The computational results of Jiang mentioned above can be used in connection with the examples of Propositions 4.3 and 4.4. In addition, if, in 4.4, $A$ is a 2-manifold, then techniques from [10] apply to the computation of the Nielsen number of a retract $f: A \rightarrow A$ of $F$.

For a final class of examples, suppose $A$ is a finite, $n$-dimensional subpolyhedron of $\mathbf{R}^{n}$. A regular neighborhood of $A$ in $\mathbf{R}^{n}$ must collapse to 
$A$ in such a way that if $x \in A$ is in the image of some $y \in \mathbf{R}^{n}-A$ under the collapse, then $x$ is in $A^{(n-1)}$, the $(n-1)$-skeleton of $A$. We can find $\varepsilon>0$ so that if $d(y, A)<\varepsilon$ then $y$ is in the regular neighborhood. Thus we have

Proposition 4.5. Let $A$ be a finite, $n$-dimensional subpolyhedron of $\mathbf{R}^{n}$. There exists $\varepsilon>0$ such that if $F: \mathbf{R}^{n} \rightarrow \mathbf{R}^{n}$ has the properties: $d(F(x), A)$ $<\varepsilon$ for all $x \in A$ and $F\left(A^{(n-1)}\right) \subseteq A$, then $F$ is retractible onto $A$.

We have arbitrarily limited the examples in this section by requiring that $X$ be a euclidean space. However, all the other results of this paper apply if $X$ is any locally finite polyhedron and some require even less. So the material in the following sections can be used in many situations not included in the preceding list of examples.

5. Deformation retractions. Now we present the example, promised in $\S 3$, that illustrates the fact that, for $F: X \rightarrow X$ retractible onto $A$, the lower bound $N(f)$ for the number of fixed points of $F$ in $A$ guaranteed by Proposition 3.3 depends on the choice of the retraction $\rho: W \rightarrow A$. Let $A$ be the unit circle centered at the origin in the plane $\mathbf{R}^{2}$ and let $W=A \cup J$ where $J$ is the simple closed curve defined, in polar coordinates, by

$$
J=\left\{\left(\cos \theta, \frac{1}{2} \sin \theta\right) \mid 0 \leq \theta \leq 2 \pi\right\} .
$$

The radial retraction of $\S 2$ restricts to a retraction $\rho_{1}: W \rightarrow A$. Let $F$ : $A \rightarrow J$ be a map such that the retract $f_{1}=\rho_{1} F_{l}: A \rightarrow A$ is homotopic to the identity and has fixed points only at $(1,0)$ and $(-1,0)$. Extend $F$ to a $\operatorname{map} F: \mathbf{R}^{2} \rightarrow \mathbf{R}^{2}$.

Let $\rho_{2}: W \rightarrow A$ be the retraction defined by first reflecting $J$ about the $x$-axis and then radially retracting it onto $A$. The retract $f_{2}=\rho_{2} F$ : $A \rightarrow A$ is a map of degree -1 . For a self-map $f$ of $A=S^{1}$, the Nielsen number $N(f)$ equals $|\operatorname{deg}(f)-1|$ (see [4; page 107]), therefore $N\left(f_{1}\right)=0$ and $N\left(f_{2}\right)=2$.

By Proposition 3.3, every self-map of $\mathbf{R}^{2}$ retractible onto $S^{1}$ so its retract is homotopic to $f_{2}$ has at least two fixed points. But 3.3 makes no such claim if the retract is homotopic to $f_{1}$ and in fact it is easy to find a self-map $F$ of $\mathbf{R}^{2}$ retractible onto $A=S^{1}$ so that its retract is homotopic to $f_{1}$ and $F$ has no fixed points.

As we mentioned in $\S 3$, we can obtain a single lower bound for the number of points of $\operatorname{Fix}(F)$ in $A$ by limiting ourselves to deformation retractions $\rho: W \rightarrow A$. If, for $j=0,1$, we have deformation retractions $\rho_{j}$ : $W \rightarrow A$ then, since $\rho_{0}$ and $\rho_{1}$ are both homotopic to the identity map of $W$, they are homotopic to each other by some homotopy $H: W \times I \rightarrow W$. 
Let $h_{t}: W \rightarrow W$ be defined by $h_{t}(x)=H(x, t)$ and suppose $h_{J}=\rho_{j}$ for $j=0,1$. Consider a homotopy $H^{\prime}: A \times I \rightarrow A$ defined by $H^{\prime}(x, t)=$ $\rho_{0} h_{t} F(x)$ and let $h_{t}^{\prime}(x)=H^{\prime}(x, t)$. For $t=0$ we have $h_{0}^{\prime}(x)=\rho_{0} h_{0} F(x)$ $=\rho_{0}\left(\rho_{0} F(x)\right)=\rho_{0} F(x)$ because $\rho_{0} F(x) \in A$. Let $f_{0}$ be the retract of $F$ with respect to $\rho_{0}$, then we have proved that $h_{0}^{\prime}=f_{0}$. Similarly $h_{1}^{\prime}=f_{1}$, the retract of $F$ with respect to $\rho_{1}$, so since homotopic maps have equal Nielsen numbers, we conclude that $N\left(f_{0}\right)=N\left(f_{1}\right)$ as we claimed in $\S 3$.

We have seen many examples of deformation retractions in previous sections, but the requirement that $\rho: W \rightarrow A$ be a deformation retraction actually represents quite a strong restriction because a deformation retraction is a homotopy equivalence, so $W$ must be the same homotopy type as $A$.

Suppose we do restrict ourselves to deformation retractions. Although it is now the single lower bound for the number of points in $\operatorname{Fix}(F)$, the Nielsen number $N(f)$ still depends on the choices of $A$ and $W$. For example, consider a rotation $F$ of the plane about the origin. If $W=A=$ $B_{R}$, as in $\S 1$, we have $N(f)=1$ while if $W=A=A_{r, R}$, as in $\S 2$, then $N(f)=0$. On the other hand, the Nielsen number of a map on a compact ANR depends only on the homotopy class of the map. We would like a Nielsen-type number for retractible maps $F: X \rightarrow X$, that is, a lower bound for the number of points in $\operatorname{Fix}(F)$ that depends only on the homotopy class of $F$ as a map from $X$ to itself.

We can obtain such a number for $F$ if we retain the restriction to deformation retractions and impose one further condition on the map $F$. One way to state this condition on $F$ is to choose $W$ and require $F(W) \subseteq W$ instead of just $F(A) \subseteq W$ as in the definition of retractibility onto $A$. However, once we require that $F$ take $W$ to itself, we have a self-map of a space (compare Remark (2) following Proposition 3.3), so we might as well just replace $W$ by $X$. That is, we consider deformation retractions $\rho: X \rightarrow A$ and put no restriction on the location of $F(A)$. Formally, we define a map $F: X \rightarrow X$ to be deformation retractible (dr) if there exists $A$, a compact ANR in $X$, and a deformation retraction $\rho$ : $X \rightarrow A$ such that $\operatorname{Fix}(f)=\operatorname{Fix}(F) \cap A$, where $f$ is the retract of $F$ with respect to $\rho$. For example, if in the hypotheses of Proposition 2.1 we replaced (0): $r \leq\|x\| \leq R$ implies $F(x) \neq 0$ by: $x \neq 0$ implies $F(x) \neq 0$, then $F$ would be a dr map on $X=\mathbf{R}^{2}-0$ provided it satisfied $(*),(* *)$ as well.

Notice that the definition of $F$ as a dr map does not specify a particular subspace $A$, in contrast to the notion of "retractible onto $A$ " in §3. Thus a dr map $F: X \rightarrow X$ might have retracts defined on different compact ANRs in $X$. 
Let $F: X \rightarrow X$ be a dr map, then we set $D(F)=N(f)$, where $f$ : $A \rightarrow A$ is any retract of $F$ with respect to a deformation retraction $\rho$ of $X$ onto a compact ANR such that $\operatorname{Fix}(f)=\operatorname{Fix}(F) \cap A$. We justify the notation, representing $D$ as just a function of $F$, by proving:

THEOREM 5.1. The definition of $D(F)$ depends only on the homotopy class of $F$.

Proof. Suppose that $F_{1}, F_{2}: X \rightarrow X$ are dr maps and that $F_{1}$ and $F_{2}$ are homotopic. Thus, for $j=1,2$ there are compact ANRs $A_{j}$ in $X$ and deformation retractions $\rho_{j}: X \rightarrow A_{j}$ such that, for $\iota_{j}: A_{j} \rightarrow X$ the inclusions, the retracts $f_{j}=\rho_{j} F_{j} \iota_{j}: A_{j} \rightarrow A_{j}$ have the property $\operatorname{Fix}\left(f_{j}\right)=\operatorname{Fix}\left(F_{j}\right)$ $\cap A_{j}$. The maps $\rho_{j}$ and $\iota_{j}$ are homotopy inverses of each other, so $f_{2}\left(\rho_{2} \iota_{1}\right)$ is homotopic to $\left(\rho_{2} \iota_{1}\right) f_{1}$ and, by the generalization in [9] of the theorem of Kiang and Jiang [23] (see also [21]), $N\left(f_{1}\right)=N\left(f_{2}\right)$. Therefore $D\left(F_{1}\right)=$ $D\left(F_{2}\right)$.

Notice, in the proof of 5.1, that although $F_{1}$ and $F_{2}$ themselves must be dr maps in order for $D\left(F_{1}\right)$ and $D\left(F_{2}\right)$ to be defined, the homotopy between them is not required to satisfy any fixed point conditions.

Theorem 5.1 implies that $D(F)$ is suitable as the proposed Nielsentype number, for dr maps:

Corollary 5.2. If $X$ is a topological space and $F: X \rightarrow X$ is a dr map, then any dr map $G: X \rightarrow X$ homotopic to $F$ has at least $D(F)$ fixed points.

If $X$ is itself a compact ANR, then by definition $D(F)$ is $N(F)$, the classical Nielsen number. The classical Nielsen theory was extended in [3] to maps $F: X \rightarrow X$ where $X$ is a noncompact ANR and $F$ is a compact map, that is, there exists a compact subset $K$ of $X$ such that $F(X) \subseteq K$. (For the particular case of $X$ a locally convex topological vector space, this extension was accomplished much earlier by Leray [24]). We will show that if $F$ is a compact, dr map on a noncompact ANR, then it is still true that $D(F)=N(F)$. We will need to refer to the definition of the Nielsen number in the setting of compact maps on ANRs, so we review it below.

First, just suppose $X$ is a topological space and $F: X \rightarrow X$ is a map. Call $x, x^{\prime} \in \operatorname{Fix}(F)$ equivalent if there is a path $c$ from $x$ to $x^{\prime}$ such that $c$ is homotopic to $F(c) \operatorname{rel}\left\{x, x^{\prime}\right\}$. (For the definition of homotopy relative to a subset, see for instance [33; page 23].) The equivalence classes of Fix $(F)$ are called the fixed point classes of $F$. The following easily proved 
observations are stated, in a more restricted setting, as Lemma 5.1 of Chapter I of [21].

LEMMA 5.3. Let $Y$ and $Z$ be topological spaces and let $\alpha: Y \rightarrow Z, \beta$ : $Z \rightarrow Y$ be maps. Then the restriction of $\alpha$ to $\operatorname{Fix}(\beta \alpha)$ is a homeomorphism onto $\operatorname{Fix}(\alpha \beta)$. Furthermore, $\mathbf{F}$ is a fixed point class of $\beta \alpha$ if and only if $\alpha(\mathbf{F})$ is a fixed point class of $\alpha \beta$.

Now let $X$ be an ANR, let $U$ be an open subset of $X$ and let $\alpha$ : $U \rightarrow X$ be a compact map such that $\{x \in U \mid \alpha(x)=x\}$ is compact. Then Granas [12] has defined a fixed point index $\operatorname{Ind}(\alpha, U)$. Of the several properties of that index, we will need to refer to the following:

Excision. If $U^{\prime}$ is an open subset of $U$ such that $\alpha(x)=x$ implies $x \in U^{\prime}$, then $\operatorname{Ind}(\alpha, U)=\operatorname{Ind}\left(\alpha, U^{\prime}\right)$.

Homotopy. If $H: U \times I \rightarrow X$ is a compact map such that the set $\{x \in U \mid H(x, t)=x$ for some $t\}$ is compact and we set $h_{t}(x)=H(x, t)$ for all $t$, then $\operatorname{Ind}\left(h_{0}, U\right)=\operatorname{Ind}\left(h_{1}, U\right)$.

Commutativity. If for $i=1,2$ we have open sets $U_{1}$ of ANRs $X_{i}$ and maps $\alpha: U_{1} \rightarrow X_{2}, \beta: U_{2} \rightarrow X_{1}$ such that $\beta \alpha: V_{1}=\alpha^{-1}\left(U_{2}\right) \rightarrow X_{1}$ is a compact map with the set $\left\{x \in V_{1} \mid \beta \alpha(x)=x\right\}$ compact then $\alpha \beta: V_{2}=$ $\beta^{-1}\left(U_{1}\right) \rightarrow X_{2}$ has the same properties and $\operatorname{Ind}\left(\beta \alpha, V_{1}\right)=\operatorname{Ind}\left(\alpha \beta, V_{2}\right)$.

A compact map $F: X \rightarrow X$ on an ANR has a finite number of fixed point classes $\mathbf{F}_{1}, \ldots, \mathbf{F}_{r}$, each a compact subset of $X$ [3; Theorem 6]. For $j=1, \ldots, r$, let $U_{j}$ be an open subset of $X$ such that $U_{j} \cap \operatorname{Fix}(F)=\mathbf{F}_{j}$. Call $\mathbf{F}_{j}$ essential if and only if $\operatorname{Ind}\left(F, U_{j}\right) \neq 0$. The Nielsen number of $F$, still denoted by $N(F)$, is the number of essential fixed point classes. The excision property implies that the definition is independent of the choice of $U_{j}$. The homotopy property of the fixed point index implies that if $F, G: X \rightarrow X$ are maps of an ANR homotopic by a homotopy which is a compact map, then $N(F)=N(G)$ (see Theorem 2 of [3] or Theorem 2 of [31]). The commutativity property of the index implies the following commutativity property of the Nielsen number for compact maps (compare Theorem 5.2 in Chapter I of [21]).

THEOREM 5.4. If $\alpha: Y \rightarrow Z$ and $\beta: Z \rightarrow Y$ are compact maps of ANRs, then $N(\beta \alpha)=N(\alpha \beta)$. 
Proof. Let $\mathbf{F}$ be a fixed point class of $\beta \alpha: Y \rightarrow Y$, then $\alpha(\mathbf{F})$ is a fixed point class of $\alpha \beta: Z \rightarrow Z$ by Lemma 5.3. Let $U^{\prime}$ be an open subset of $Z$ such that $U^{\prime} \cap \operatorname{Fix}(\alpha \beta)=\alpha(\mathbf{F})$ and let $U=\alpha^{-1}\left(U^{\prime}\right)$. Using Lemma 5.3, we can see that $U \cap \operatorname{Fix}(\beta \alpha)=\mathbf{F}$. By the commutativiy property of the fixed point index, $\operatorname{Ind}(\beta \alpha, U)=\operatorname{Ind}\left(\alpha \beta, V^{\prime}\right)$ where $V^{\prime}=\beta^{-1}(U)$. Since $V^{\prime} \cap \operatorname{Fix}(\alpha \beta)=\alpha(\mathbf{F})$, it follows that $\mathbf{F}$ is essential if and only if $\alpha(\mathbf{F})$ is essential. Lemma 5.3 establishes a one-to-one correspondence between the fixed point classes of $\beta \alpha$ and of $\alpha \beta$, so we conclude that $N(\beta \alpha)=$ $N(\alpha \beta)$.

We can now prove the result promised earlier.

TheOREM 5.5. Let $X$ be an ANR and let $F: X \rightarrow X$ be a compact, $d r$ map, then $D(F)=N(F)$.

Proof. Since $F$ is a dr map, there is a compact ANR $A$ in $X$ and a deformation retraction $\rho: X \rightarrow A$ such that $\operatorname{Fix}(f)=\operatorname{Fix}(F) \cap A$ for $f=\rho F \iota: A \rightarrow A$, where $\iota: A \rightarrow X$ is inclusion. The definition of deformation retraction gives us a homotopy $H: X \times I \rightarrow X$ such that $H(x, 0)=x$ and $H(x, 1)=\rho(x)$ for all $x \in X$. Noting that $\rho=\imath \rho$, we see that the compact map $F H: X \times I \rightarrow X$ is a homotopy between $F$ and $F \iota \rho$, so $N(F)=N(F \iota \rho)$. The maps $\rho: X \rightarrow A$ and $F_{\iota}: A \rightarrow X$ are compact and therefore $N(F \iota \rho)=N(\rho F \iota)=N(f)$ by Theorem 5.4. Finally $N(f)=$ $D(F)$ by definition.

6. Extensions without additional fixed points. The Minimality Theorem of $\S 7$ will state that, under sufficiently strong hypotheses, given a map $F: X \rightarrow X$ retractible onto $A$ with retract $f: A \rightarrow A$, there exists a map $G: X \rightarrow X$ retractible onto $A$, with a retract $g: A \rightarrow A$ homotopic to $f$, such that $G$ has exactly $N(f)$ fixed points. Thus the lower bound on the number of fixed points promised by Proposition 3.2 is seen to be sharp under these hypotheses.

The most general minimality theorem of this sort for maps of finite polyhedra is due to Boju Jiang [19]. We will require $A$ to be a polyhedron satisfying the hypotheses of Jiang's theorem and homotope $f$ to a map $g$ with exactly $N(f)$ fixed points. We would then like to use techniques of Gen-hua Shi in [32] to extend $g$ to a map $G: X \rightarrow X$ which has no fixed points on $X-A$ and therefore satisfies our requirements. However, we cannot use Shi's techniques without modification. Shi was concerned with producing a fixed point free map in a given homotopy class, not with extending a map already defined on a subspace. His methods will tend to change the map on $A$, in an uncontrollable way, as they extend the 
definition over all of $X$. Therefore we will devote this section to a "relative to $A$ " version of Shi's techniques.

The principal modification concerns Shi's improvement of a theorem of Hopf. Hopf proved [15] that any map on a finite polyhedron $X$ can be approximated by one whose fixed points are isolated and all lie in maximal simplices of $X$. Shi [32] extended Hopf's result to locally finite polyhedra. Our relative form of the Hopf-Shi theorem requires that the approximation be identical to the original map on a finite subpolyhedron A.

THEOREM 6.1. Let $X$ be a locally finite polyhedron, $A$ a finite subpolyhedron, and $G_{0}: X \rightarrow X$ a map such that $G_{0}(A) \subseteq A$, then there exists $a$ map $G: X \rightarrow X$ homotopic to $G_{0} \operatorname{rel} A$ (so, in particular $G(a)=G_{0}(a)$ for all $a \in A$ ) such that each fixed point of $G$ in $X-A$ is isolated and lies in $a$ maximal simplex.

Proof. Let $G_{0} \mid A: A \rightarrow A$ denote the restriction of $G_{0}$ to $A$ and set $g=G_{0} \mid A$. Let $d$ be the barycentric metric on $X$. We first show that there exists $\varepsilon>0$ and a map $G_{1}: X \rightarrow X$ such that $G_{1} \mid A=g$ and if $d(x, A) \leq \varepsilon$, then $G_{1}(x) \in A$. Since $A$ is an ANR, there is an open subset $V$ of $X$ containing $A$ and a map $G_{1}: V \rightarrow A$ such that $G_{1} \mid A=g$. Let $S(A)$ be the simplicial neighborhood of $A$ in $X$, that is, the union of all closed simplices of $X$ that intersect $A$ (see page 32 of [30]). Then $A$ is in the interior of $S(A)$ and, since $A$ is finite and $X$ is locally finite, $S(A)$ is finite. Therefore, there exists $\eta>0$ such that if $Y$ is any space and $a, b$ : $Y \rightarrow S(A)$ are maps with $d(a(y), b(y))<\eta$ for all $y \in Y$, then $a$ and $b$ are homotopic by a homotopy that is constant at $y$ if $a(y)=b(y)$ (see the proof of Theorem 1.1 on page 111 of [16]). Let int $S(A)$ denote the interior of $S(A)$ and let $W=G_{0}^{-1}$ (int $S(A)$ ). Then $W$ is a neighborhood of $A$ in $X$ and it follows that $d(A, X-(V \cap W))>0$. Subdivide $S(A)$ so that the mesh of the triangulation is less than $d(A, X-(V \cap W))$. Let $S^{\prime}(A)$ be the simplicial neighborhood of $A$ in $S(A)$ with respect to this triangulation, then $S^{\prime}(A) \subseteq V \cap W$. Now $G_{0}$ and $G_{1}$ are uniformly continuous on $S^{\prime}(A)$ so, for $i=0,1$, there exist $\varepsilon_{i}>0$ such that if $x, x^{\prime} \in$ $S^{\prime}(A)$ and $d\left(x, x^{\prime}\right)<\varepsilon_{i}$, then $d\left(G_{i}(x), G_{i}\left(x^{\prime}\right)\right)<\eta / 2$. Let

$$
2 \varepsilon=\min \left\{\varepsilon_{0}, \varepsilon_{1}, d\left(A, X-\operatorname{int} S^{\prime}(A)\right)\right\}
$$

and suppose $x \in X$ with $d(x, A) \leq \varepsilon$. There exists $x^{\prime} \in A$ such that $d\left(x, x^{\prime}\right)=d(x, A)$. Keeping in mind that $G_{0}\left|A=G_{1}\right| A=g$, we see that

$$
\begin{aligned}
d\left(G_{0}(x), G_{1}(x)\right) & \leq d\left(G_{0}(x), g\left(x^{\prime}\right)\right)+d\left(g\left(x^{\prime}\right), G_{1}(x)\right) \\
& =d\left(G_{0}(x), G_{0}\left(x^{\prime}\right)\right)+d\left(G_{1}\left(x^{\prime}\right), G_{1}(x)\right)<\eta .
\end{aligned}
$$


Let

$$
\bar{N}_{\varepsilon}(A)=\{x \in X \mid d(x, A) \leq \varepsilon\}
$$

then $\bar{N}_{\varepsilon}(A) \subseteq S^{\prime}(A) \subseteq V \cap W$. Since $G_{0}(W) \subseteq S(A)$ and $G_{1}(V) \subseteq A \subseteq$ $S(A)$, then $G_{1} \mid \bar{N}_{\varepsilon}(A)$ and $G_{2} \mid \bar{N}_{\varepsilon}(A)$ are homotopic in $S(A) \subseteq X$ rel $A$. Since $X$ is an ANR and $G_{0} \mid \bar{N}_{\varepsilon}(A)$ extends to $G_{0}: X \rightarrow X$ then $G_{1} \mid \bar{N}_{\varepsilon}(A)$ extends to $G_{1}: X \rightarrow X$ homotopic to $G_{0}$ rel $A$. Now $G_{1}\left(\bar{N}_{\varepsilon}(A)\right) \subseteq G_{1}(V)$ $\subseteq A$ so $G_{1}$ has the required property.

Next subdivide $S^{\prime}(A)$ to obtain a triangulation of mesh less than $\varepsilon / 4$, and extend arbitrarily to a triangulation of $X$. Define $N_{\varepsilon / 2}(A)=\{x \in$ $X \mid d(x, A)<\varepsilon / 2\}$. Let $K$ be the subpolyhedron of $X$ consisting of all closed simplices of $X$ (with respect to the new triangulation) that intersect $X-N_{\varepsilon / 2}(A)$. Let $G_{1}^{\prime}=G_{1} \mid K: K \rightarrow X$, then $G_{1}^{\prime}$ is a map of simplicial complexes so there is a subdivision of $K$ and a simplicial approximation $\varphi: K \rightarrow X$ of $G_{1}^{\prime}$ with respect to that triangulation. We will need the map $t: \bar{N}_{\varepsilon}(A) \rightarrow \mathbf{R}$ (the reals) defined by

$$
t(x)=\frac{4 d(x, A)}{\varepsilon}-2 .
$$

Define $G_{2}: X \rightarrow X$ as follows

$$
G_{2}(x)= \begin{cases}G_{1}(x) & \text { if } d(x, A) \leq \varepsilon / 2 \\ (1-t(x)) G_{1}(x)+t(x) \varphi(x) & \text { if } \varepsilon / 2 \leq d(x, A) \leq 3 \varepsilon / 4 \\ \varphi(x) & \text { if } d(x, A) \geq 3 \varepsilon / 4\end{cases}
$$

Clearly $G_{2}$ is homotopic to $G_{1}$ because $\varphi$ is homotopic to $G_{1}^{\prime}$. Note that if $\varepsilon / 2 \leq d(x, A) \leq \varepsilon$ then $x \in K$ so $\varphi(x)$ is defined and, since $x \in \bar{N}_{\varepsilon}(A)$, then we know $G_{1}(x) \in A$. Since $\varphi$ is a simplicial approximation to $G_{1}^{\prime}$, then there is a simplex containing both $\varphi(x)$ and $G_{1}(x)$, so that simplex will intersect $A$. Since the simplex is in $S^{\prime}(A)$, its diameter is less than $\varepsilon / 4$. We conclude that if $\varepsilon / 2 \leq d(x, A) \leq \varepsilon$, then $d\left(G_{2}(x), A\right)<\varepsilon / 4$ and, in particular, $G_{2}(x) \neq x$. If $0<d(x, A) \leq \varepsilon / 2$ then $G_{2}(x)=G_{1}(x) \in A$ so $G_{2}$ has no fixed points on $\bar{N}_{\varepsilon}(A)-A$. Now $G_{2}$ is simplicial on a subcomplex of $K$ containing $X-\bar{N}_{\varepsilon}(A)$ so by the first part of the proof of Lemma 3 of [32], all the fixed points of $G_{2}$ on $X-A$ are isolated. For the rest of the proof we just copy Shi's argument for Lemma 3, always choosing $\delta_{i}<\varepsilon / 4$ so all homotopies are relative to $A$ (in fact to $N_{\varepsilon / 2}(A)$ ). Thus we obtain a map $G: X \rightarrow X$ with the required properties.

A polyhedron $X$ is said to be two-dimensionally connected if for any two maximal simplices $\sigma$ and $\tau$ of $X$ there are simplices $\sigma=\sigma_{0}$, 
$\sigma_{1}, \ldots, \sigma_{n-1}, \sigma_{n}=\tau$ such that $\sigma_{i-1}$ and $\sigma_{i}$ have a common face of dimension at least one, for $i=1,2, \ldots, n$.

Theorem 3 of [32] states that if $X$ is an infinite, locally finite, and two-dimensionally connected polyhedron, then any map $G: X \rightarrow X$ is homotopic to a fixed point free map. As explained above, we require a "relative to $A$ " version.

Note that if $G: X \rightarrow X$ is a map such that $G(A) \subseteq A$, then $G$ is (trivially) retractible onto $A$ and its retract $g: A \rightarrow A$ is just the restriction of $G$ to $A$.

THEOREM 6.2. Suppose $X$ is a locally finite polyhedron and $A$ is a finite subpolyhedron such that each component of $X-A$ is infinite and two-dimensionally connected. If $F: X \rightarrow X$ is a map such that $G(A) \subseteq A$, then there exists a map $G^{\prime}: X \rightarrow X$ homotopic to $G \operatorname{rel} A$ such that $\operatorname{Fix}\left(G^{\prime}\right)=\operatorname{Fix}(G \mid A)$.

Proof. By Theorem 6.1, we assume that each fixed point of $G$ in $X-A$ is isolated and in a maximal simplex. We may apply the proof of Theorem 1 of [32] to each component of $X-A$ to obtain the map $G^{\prime}$, if we make the following observations. Let $U$ be a component of $X-A$. The one-complex $R$ of Shi's proof has vertices in a one-to-one correspondence, all the correspondence $\psi$, with the maximal simplexes of $U$. For $\sigma_{1}, \sigma_{2}$ maximal simplices of $U$, we will require that the vertices $\psi\left(\sigma_{1}\right), \psi\left(\sigma_{2}\right)$ of $R$ form a one-simplex of $R$ if, and only if, $\sigma_{1}$ and $\sigma_{2}$ have a common face in $U$ of dimension at least one. The hypothesis that $U$ is two-dimensionally connected implies that $R$ is connected. Then, in the last part of the proof of Shi's Theorem 1, when his Lemma 1 is used, the definition of $R$ above assures us that the homotopy is relative to the boundary of $U$, which is a subset of $A$.

Theorem 6.2 has some consequences that are of interest in themselves, independent of the minimality theorem.

COROLlary 6.3. Suppose $X$ is a locally finite polyhedron and $A$ is a finite subpolyhedron such that each component of $X-A$ is infinite and two-dimensionally connected. If $G: X \rightarrow X$ is a map such that $G(A) \subseteq A$ and the restriction $g: A \rightarrow A$ is fixed point free, then there exists a fixed point free map $G^{\prime}: X \rightarrow X$ homotopic to $G \operatorname{rel} A$.

COROLlaRY 6.4. Let $A$ be a finite polyhedron in the euclidean space $\mathbf{R}^{n}$ such that $\mathbf{R}^{n}-A$ is connected. Given a fixed point free map $g: A \rightarrow A$, there exists a fixed point free map $G: \mathbf{R}^{n} \rightarrow \mathbf{R}^{n}$ extending $g$.

Proof. The hypothesis that $\mathbf{R}^{n}-A$ is connected forces $n \geq 2$ so $\mathbf{R}^{n}-A$ is an open $n$-manifold, $n \geq 2$, and therefore is two-dimensionally 
connected. Since $\mathbf{R}^{n}$ has the absolute extension property, there is a map $G^{\prime}: \mathbf{R}^{n} \rightarrow \mathbf{R}^{n}$ extending $g$. Now apply Theorem 6.2 .

To see that we must require that $\mathbf{R}^{n}-A$ be connected, consider the antipodal map $g$ on the unit circle in $\mathbf{R}^{2}$. Any extension of $g$ to $\mathbf{R}^{2}$ produces a map of the unit disc into $\mathbf{R}^{2}$ taking the boundary back into the disc. Such a map must have a fixed point by the Poincaré-Bohl Theorem (Theorem 1.2).

7. The Minimality Theorem. A point $x$ in a space $X$ is a local separating ( = cut ) point if there is a connected neighborhood $U$ of $x$ in $X$ such that $U-\{x\}$ is disconnected.

Let $X$ be a space and let $A$ be a subspace of $X$. A map $f: A \rightarrow A$ is $X$-extendable if there exists a map $F^{\prime}: X \rightarrow X$ such that $F^{\prime} \mid A=f$.

The term "extendable" is sometimes used by itself for the concept we have called $X$-extendable [11; page 176], [25; page 22]. However, since, for example, the identity map on the unit circle is extendable over the plane as a map into the plane but not as a map into the circle, it is important to specify the range of the extension (compare [7; page 13]).

Proposition 7.1. Suppose $X$ is an ANR and $A$ is a subset of $X$. Each of the following three conditions is sufficient for a map $f: A \rightarrow A$ to be $X$-extendable:

(i) $A$ is a retract of $X$;

(ii) $X$ is contractible;

(iii) $f=\rho F \iota: A \rightarrow A$ is a retract of a map $F: X \rightarrow X$ and $\rho: X \rightarrow A$ is a deformation retraction.

Proof. (i) Let $r: X \rightarrow A$ be the retraction and set $F^{\prime}=\iota$ fr: $X \rightarrow X$ where $\iota: A \rightarrow X$ is inclusion. (ii) Since $X$ is an absolute retract, it is an absolute extensor so $\iota f: A \rightarrow X$ extends to a map $F^{\prime}: X \rightarrow X$. (iii) Of course $F: A \rightarrow X$ extends to $F: X \rightarrow X$. Since $\rho$ is a deformation retraction, $\iota f=\imath \rho F \iota: A \rightarrow X$ is homotopic to $F \iota$. Now $X$ is an ANR, so $f$ extends to a map $F^{\prime}: X \rightarrow X$ homotopic to $F$ by the Homotopy Extension Theorem.

EXAMPle. We will show that part (iii) of Proposition 7.1 fails if $\rho$ is assumed only to be a retraction of the sort we used in $\S 3$, rather than a deformation retraction of $X$ onto $A$. Let $e^{m}$ denote a closed $m$-cell. As a spherical complex, the quaternionic projective plane $\mathbf{H} P^{2}$ can be written in the form $e^{0} \cup e^{4} \cup e^{8}$ (we omit the attaching maps from our notation). The 4-sphere $e^{0} \cup e^{4}$ generates $H^{4}\left(\mathbf{H} P^{2} ; \mathbf{Z}\right)$. Let $X=\mathbf{H} P^{2} \times \mathbf{R}$ and 
$A=\left(e^{0} \cup e^{4}\right) \times\{0\}$, then define $F: X \rightarrow X$ by $F(q, t)=(q, t+1)$ for $q \in \mathbf{H} P^{2}, t \in \mathbf{R}$. Identify $\left(e^{0} \cup e^{4}\right) \times\{t\}$ with $S^{4} \subseteq \mathbf{R}^{5}$ for each $t \in \mathbf{R}$ and define a retraction

$$
\rho:\left(e^{0} \cup e^{4}\right) \times\{0,1\}=A \cup F(A) \rightarrow A
$$

by $\rho(q, t)=\left((-1)^{t} q, 0\right)$. Then $F$ is retractible onto $A$ with the retract defined by $f(q, 0)=(-q, 0)$, which is of degree -1 on $A$. Suppose $f$ were $X$-extendable to a map $F^{\prime}: X \rightarrow X$, then $F^{\prime}$ induces

$$
F^{*}: H^{4}(X ; \mathbf{Z}) \rightarrow H^{4}(X ; \mathbf{Z}) \cong H^{4}\left(\mathbf{H} P^{2} ; \mathbf{Z}\right)
$$

and $F^{*}(\omega)=-\omega$ for $\omega$ a generator of $H^{4}(X ; \mathbf{Z})$. But a Steenrod algebra argument due to Glen Bredon (see [8; page 192]) shows that such a map $F^{\prime}$ cannot exist.

We come now to the main result of this section.

Theorem 7.2 (Minimality Theorem.) Suppose $X$ is a locally finite polyhedron and $A$ is a finite subpolyhedron without local separating points, but not a surface of negative Euler characteristic, such that each component of $X-A$ is infinite and two-dimensionally connected. If a map $F: X \rightarrow X$ is retractible to an $X$-extendable map $f: A \rightarrow A$, then there exists a map $G$ : $X \rightarrow X$ retractible to a map $g: A \rightarrow A$ homotopic to $f$ such that $G$ has exactly $N(f)$ fixed points.

Proof. By Theorem 5.3 of [19], there exists a map $g: A \rightarrow A$ homotopic to $f$ such that $g$ has exactly $N(f)$ fixed points. Since $f$ is $X$-extendable and $X$ is an ANR, the Homotopy Extension Theorem permits us to extend $g$ to a map $G^{\prime}: X \rightarrow X$. Theorem 6.2 completes the argument by producing the required map $G$ on $X$. Notice that $G(A)=G^{\prime}(A)=g(A)$ $\subseteq A$ so $G$ is, trivially, retractible onto $A$.

The next result furnishes many examples of the use of the Minimality Theorem.

THEOREM 7.3. Let $A$ be a compact triangulated n-manifold with nonempty boundary in $\mathbf{R}^{n}, n \geq 3$, such that $A$ has a spine of dimension less than $n-1$. If a map $F: \mathbf{R}^{n} \rightarrow \mathbf{R}^{n}$ is retractible onto $A$ with retract $f: A \rightarrow A$, then there exists a map $G: \mathbf{R}^{n} \rightarrow \mathbf{R}^{n}$ retractible to a map $g: A \rightarrow A$ homotopic to $f$ such that $G$ has exactly $N(f)$ fixed points.

Proof. Since $A$ is an $n$-manifold, $n \geq 3$, it is not a surface and it has no local separating points. Its complement $X-A$ is connected by 
Alexander duality [33; page 296] and it is an open $n$-manifold, so it is two-dimensionally connected. The map $f$ is $\mathbf{R}^{n}$-extendable by part (ii) of Proposition 7.1.

Propositions 4.1, 4.2 and 4.3 all provide examples of maps satisfying the hypotheses of Theorem 7.3. For instance,

COROLlaRy 7.4. Let $A$ be a compact $n$-manifold, $n \geq 7$, with nonempty boundary $\partial A$ such that $\pi_{i}(A, \partial A)=0$ for all $i \leq 3$ and suppose $A$ is embedded in $\mathbf{R}^{n}$ as a subpolyhedron. Let $Y$ be a complementary spine of $A$ in $S^{n}=\mathbf{R}^{n} \cup\{\infty\}$ with $\infty \in Y$. If $F: \mathbf{R}^{n} \rightarrow \mathbf{R}^{n}$ is a map such that $F(A) \cap Y$ $=\varnothing$ and $F(\partial A) \subseteq A$, then every map retractible onto $A$ with retract homotopic to $f: A \rightarrow A$, the retract of $F$ with respect to a retraction of $\mathbf{R}^{n}-Y$ onto $A$, has at least $N(f)$ fixed points and one such map has exactly $N(f)$ fixed points.

Proof. The homotopy condition implies that $A$ has a spine of dimension less than $n-1$ [34]. The map $F$ is retractible onto $A$ by Proposition 4.2. Proposition 3.3 and Theorem 7.3 complete the proof.

In the setting of the dr maps of $\S 5$, the Minimality Theorem has the following form.

Theorem 7.5. Suppose $X$ is a locally finite polyhedron and $F: X \rightarrow X$ is $a d r$ map. Suppose also that $F$ has a retract $f: A \rightarrow A$ with respect to a deformation retraction and $\operatorname{Fix}(f)=\operatorname{Fix}(F) \cap A$, where $A$ is a finite subpolyhedron of $X$ with no local separating points, but $A$ is not a surface of negative Euler characteristic, and such that each component of $X-A$ is infinite and two-dimensionally connected. Then there exists a $d r$ map $G$ : $X \rightarrow X$ homotopic to $F$ such that $G$ has exactly $D(F)$ fixed points.

Proof. Any retract of $F$ with respect to a deformation retraction is $X$-extendable by part (iii) of Proposition 7.1. Furthermore, any such extension is homotopic to $F$. The required map $G$ is produced by Theorem 7.2 if we note that by using the full statement of Theorem 6.2 in the proof we obtain a map homotopic to $F$. The map $G$ is dr because $G(A) \subseteq A$ so we may use the deformation retraction of $X$ onto $A$, that we know exists because $F$ is a dr map, to satisfy the same requirement for $G$.

The material of $\$ 4$ can again supply examples, now of maps satisfying the hypotheses of Theorem 7.5. For instance, corresponding to Proposition 4.1 we have 
Corollary 7.6. Let $A=A(k, \varepsilon)$ be a solid torus in $\mathbf{R}^{n}$ and let $X=\left\{\left(x_{1}, \ldots, x_{n}\right) \in \mathbf{R}^{n} \mid x_{1}^{2}+x_{2}^{2} \neq 0\right\}$. If a map $F: X \rightarrow X$ is weakly inward on $\partial A$, then $F$ is a dr map and there exists a dr map $G: X \rightarrow X$ homotopic to $F$ such that $G$ has exactly $D(F)$ fixed points.

Finally, the Minimality Theorem gives us the following result, related to Corollary 6.3, concerning fixed point free maps.

COROLlary 7.7. Suppose $X$ is a locally finite polyhedron and $A$ is a finite subpolyhedron without local separating points, but not a surface with negative Euler characteristic, such that each component of $X-A$ is infinite and two-dimensionally connected. If a map $F: X \rightarrow X$ is retractible to an $X$-extendable map $f: A \rightarrow A$ with $N(f)=0$ (equivalently, $f$ is homotopic to a fixed point free map), then there exists a fixed point free map $G: X \rightarrow X$ such that $G(A) \subseteq A$ and $g$, the restriction of $G$ to $A$, is homotopic to $f$.

\section{REFERENCES}

[1] P. Bohl, Über die Bewegung eines mechanischen Systems in der Nähe einer Gleichgewichtslage, Journal für Math., 127 (1904), 179-200.

[2] L. Brouwer, Über die Minimalzahl der Fixpunkte bei den Klassen von eindeutigen stetigen Transformationen der Ringflächen, Math. Ann., 82 (1921), 94-96.

[3] R. Brown, On the Nielsen fixed point theorem for compact maps, Duke Math J., 36 (1969), 699-708.

[4] The Lefschetz Fixed Point Theorem, Scott-Foresman, 1971.

[5] _ Multiple fixed points of compact maps on wedgelike ANRs in Banach spaces, Topological Methods in Nonlinear Functional Analysis, Contemporary Math., Vol. 21, 1983, 41-57.

[6] J. Caristi, Fixed point theorems for mappings satisfying inwardness conditions, Trans. Amer. Math. Soc., 215 (1976), 241-251.

[7] J. Dugundji, Topology, Allyn and Bacon, 1966.

[8] E. Dyer, Some remarks on fixed point theory, General Topology and Modern Analysis, Academic Press, 1981, 185-208.

[9] E. Fadell, Nielsen numbers as homotopy type invariants, Pacific J. Math., 63 (1976), 381-388.

[10] E. Fadell and S. Husseini, The Nielsen number on surfaces, to appear.

[11] S. Gaal, Point Set Topology, Academic Press, 1964.

[12] A. Granas, The Leray-Schauder index and the fixed point theory for arbitrary ANRs, Bull. Soc. Math. France, 100 (1972), 209-228.

[13] M. Hadamard, Note sur quelques applications de l'indice de Kronecker, in J. Tannery, Introduction a la Théorie des Fonctions, Vol. 2, Hermann, 1910, 437-477.

[14] H. Hopf, Über Mindestzahl von Fixpunkten, Math. Z., 26 (1927), 762-774.

[15] __ Über die algebraische Anzahl von Fixpunkten, Math. Z., 29 (1929), 493-524.

[16] S. Hu, Theory of Retracts, Wayne State Univ., 1965.

[17] J. Hudson, Piecewise Linear Topology, Benjamin, 1969.

[18] B. Jiang, Estimation of the Nielsen numbers, Chinese Math., 5 (1964), 330-339. 
[19] On the least number of fixed points, Amer. J. Math., 102 (1980), 749-763.

[20] __, On the computation of the Nielsen numbers, Pacific J. Math., to appear.

[21] _ Lectures on Nielsen Fixed Point Theory, Contemporary Math., Vol. 14, 1983.

[22] S. Kaniel, Quasi-compact non-linear operators in Banach space and applications, Arch. Rational Mech. Anal., 20 (1965), 259-278.

[23] T. Kiang and B. Jiang, The Nielsen number of self-mappings of the same homotopy type, Sci. Sinica, 12 (1963), 1071-1072.

[24] J. Leray, Le théorie des points fixes et ses applications in analyse, Proc. International Congress of Math., 1950, Vol. 2, Amer. Math. Soc., 1952, 202-208.

[25] G. McCarty, Topology, McGraw-Hill, 1967.

[26] J. Munkres, Elementary Differential Topology, Princeton Univ., 1966.

[27] J. Nielsen, Über die Minimalzahl der Fixpunkte bei den Abbildungstypen der Ringflächen, Math. Ann., 82 (1921), 83-93.

[28] H. Poincaré, Sur les courbes définies par les équations differentielles, IV, Journal de Math. (4), 2 (1886), 151-217.

[29] C. Rourke, A note on complementary spines, Proc. Camb. Philos. Soc., 63 (1967), 1-3.

[30] C. Rourke and B. Sanderson, Introduction to Piecewise-Linear Topology, Springer, 1972.

[31] K. Scholz, The Nielsen fixed point theory for noncompact spaces, Rocky Mt. J. Math., 4 (1974), 81-87.

[32] G. Shi, On the least number of fixed points for infinite complexes, Pacific J. Math., to appear.

[33] E. Spanier, Algebraic Topology, McGraw-Hill, 1966.

[34] C. Wall, Geometrical connectivity I, J. London Math. Soc., 3 (1971), 597-604.

Received February 14, 1983 and in revised form August 24, 1983.

UNIVERSITY OF CALIFORNIA, LOS ANGELES

LOS ANGELES, CA 90024 



\title{
PACIFIC JOURNAL OF MATHEMATICS EDITORS
}

\author{
Donald BABBITt (Managing Editor) \\ University of California \\ Los Angeles, CA 90024 \\ J. DugunduI \\ University of Southern California \\ Los Angeles, CA 90089-1113 \\ R. FINN \\ Stanford University \\ Stanford, CA 94305 \\ HermanN FlaschKa \\ University of Arizona \\ Tucson, AŻ 85721
}

C. C. Moore

University of California

Berkeley, CA 94720

ARTHUR Ogus

University of California

Berkeley, CA 94720

Hugo Rossi

University of Utah

Salt Lake City, UT 84112

H. SAMELSON

Stanford University

Stanford, CA 94305

ASSOCIATE EDITORS
R. ARENS
E. F. BECKENBACH
B. H. NEUMANN
F. WOLF
K. YoSHIDA (1906-1982)

\section{SUPPORTING INSTITUTIONS}

$\begin{array}{ll}\text { UNIVERSITY OF ARIZONA } & \text { UNIVERSITY OF OREGON } \\ \text { UNIVERSITY OF BRITISH COLUMBIA } & \text { UNIVERSITY OF SOUTHERN CALIFORNIA } \\ \text { CALIFORNIA INSTITUTE OF TECHNOLOGY } & \text { STANFORD UNIVERSITY } \\ \text { UNIVERSITY OF CALIFORNIA } & \text { UNIVERSITY OF HAWAII } \\ \text { MONTANA STATE UNIVERSITY } & \text { UNIVERSITY OF TOKYO } \\ \text { UNIVERSITY OF NEVADA, RENO } & \text { UNIVERSITY OF UTAH } \\ \text { NEW MEXICO STATE UNIVERSITY } & \text { WASHINGTON STATE UNIVERSITY } \\ \text { OREGON STATE UNIVERSITY } & \text { UNIVERSITY OF WASHINGTON }\end{array}$

The Supporting Institutions listed above contribute to the cost of publication of this Journal, but they are not owners or publishers and have no responsibility for its content or policies.

Mathematical papers intended for publication in the Pacific Journal of Mathematics should be in typed form or offset-reproduced (not dittoed), double spaced with large margins. Please do not use built up fractions in the text of the manuscript. However, you may use them in the displayed equations. Underline Greek letters in red, German in green, and script in blue. The first paragraph must be capable of being used separately as a synopsis of the entire paper. In particular it should contain no bibliographic references. Please propose a heading for the odd numbered pages of less than 35 characters. Manuscripts, in triplicate, may be sent to any one of the editors. Please classify according to the scheme of Math. Reviews, Index to Vol. 39. Supply name and address of author to whom proofs should be sent. All other communications should be addressed to the managing editor, or Elaine Barth, University of California, Los Angeles, California 90024.

There are page-charges associated with articles appearing in the Pacific Journal of Mathematics. These charges are expected to be paid by the author's University, Government Agency or Company. If the author or authors do not have access to such Institutional support these charges are waived. Single authors will receive 50 free reprints; joint authors will receive a total of 100 free reprints. Additional copies may be obtained at cost in multiples of 50 .

The Pacific Journal of Mathematics is issued monthly as of January 1966. Regular subscription rate: $\$ 190.00$ a year (5 Vols., 10 issues). Special rate: $\$ 66.00$ a year to individual members of supporting institutions.

Subscriptions, orders for numbers issued in the last three calendar years, and changes of address should be sent to Pacific Journal of Mathematics, P.O. Box 969, Carmel Valley, CA 93924, U.S.A. Old back numbers obtainable from Kraus Periodicals Co., Route 100, Millwood, NY 10546.

The Pacific Journal of Mathematics at P.O. Box 969, Carmel Valley, CA 93924 (ISSN 0030-8730) publishes 5 volumes per year. Application to mail at Second-class postage rates is pending at Carmel Valley, California, and additional mailing offices. Postmaster: Send address changes to Pacific Journal of Mathematics, P.O. Box 969, Carmel Valley, CA 93924.

PUBLISHED BY PACIFIC JOURNAL OF MATHEMATICS, A NON-PROFIT CORPORATION

Copyright $@ 1984$ by Pacific Journal of Mathematics 


\section{Pacific Journal of Mathematics}

\section{Vol. 115, No. $2 \quad$ October, 1984}

Ersan Akyildiz, Gysin homomorphism and Schubert calculus ...........257

Marilyn Breen, Clear visibility and unions of two starshaped sets in the

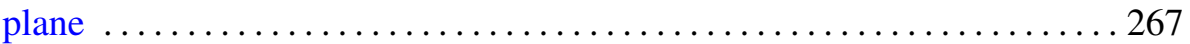

Robert F. Brown, Retraction methods in Nielsen fixed point theory . ......277

Herbert Busemann and Bhalchandra B. Phadke, A general version of Beltrami's theorem in the large ............................... 299

Gerald Arthur Edgar and Robert Francis Wheeler, Topological properties of Banach spaces ............................... 317

Yaakov Friedman and Bernard Russo, Conditional expectation without

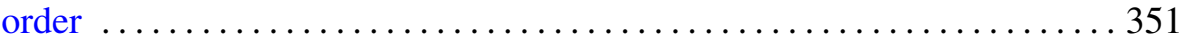

Robert Allen Goggins, Cobordism of manifolds with strong almost tangent

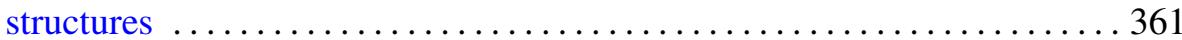

Mike Hoffman, Noncoincidence index of manifolds . . . . . . . . . . . . . 373

William H. Julian, $\varepsilon$-continuity and monotone operations $\ldots \ldots \ldots \ldots 385$

Gerasimos E. Ladas, Y. G. Sficas and I. P. Stavroulakis, Nonoscillatory

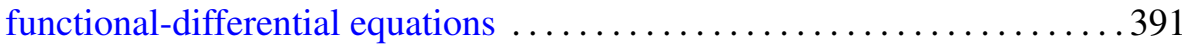

Arnold William Miller and Karel Libor Prikry, When the continuum has

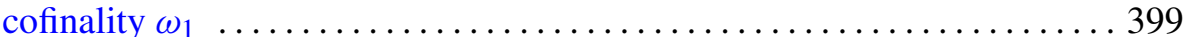

Jean-Leah Mohrherr, Density of a final segment of the truth-table

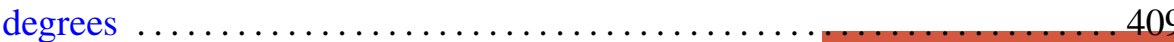

Carl Norman Mutchler, The flat Cauchy problem for radially hyperbolic operators from a characteristic manifold of high codimension ...

Kenji Nakagawa, On the orders of automorphisms of a closed Riemann surface

W. Ricker, Representation of vector-valued functions by Laplace transforms

Jorge Donato Samur, On semigroups of convolution operators in Hilbert

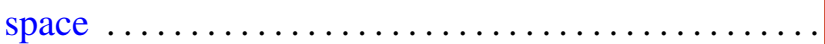

Joseph Gail Stampfli, One-dimensional perturbations of operators 481 Andrew George Earnest and John Sollion Hsia, Correction to: "Spinor norms of local integral rotations. II" 